\title{
Unsupervised Assessment of Subcutaneous and Visceral Fat by MRI
}

\author{
Peter S. Jørgensen ${ }^{1,2}$, Rasmus Larsen ${ }^{1}$, and Kristian Wraae ${ }^{3}$ \\ 1 Department of Informatics and Mathematical Modelling, \\ Technical University of Denmark, Denmark \\ 2 Fuel Cells and Solid State Chemistry Division, National Laboratory for Sustainable \\ Energy, Technical University of Denmark, Denmark \\ 3 Odense University Hospital, Denmark
}

\begin{abstract}
This paper presents a method for unsupervised assessment of visceral and subcutaneous adipose tissue in the abdominal region by MRI. The identification of the subcutaneous and the visceral regions were achieved by dynamic programming constrained by points acquired from an active shape model. The combination of active shape models and dynamic programming provides for a both robust and accurate segmentation. The method features a low number of parameters that give good results over a wide range of values. The unsupervised segmentation was compared with a manual procedure and the correlation between the manual segmentation and unsupervised segmentation was considered high.
\end{abstract}

Keywords: Image processing, Abdomen, Visceral fat, Dynamic programming, Active shape model.

\section{Introduction}

There is growing evidence that obesity is related to a number of metabolic disturbances such as diabetes and cardiovascular disease [1]. It is of scientific importance to be able to accurately measure both visceral adipose tissue (VAT) and subcutaneous adipose tissue (SAT) distributions in the abdomen. This is due to the metabolic disturbances being closely correlated with particularly visceral fat 2 .

Different techniques for fat assessment is currently available including anthropometry (waist-hip ratio, Body Mass Index), computed tomography (CT) and magnetic resonance imaging (MRI) 3 .

These methods differ in terms of cost, reproducibility, safety and accuracy. The anthropometric measures are easy and inexpensive to obtain but do not allow quantification of visceral fat. Other techniques like CT will allow for this distinction in an accurate and reproducible way but are not safe to use due to the ionizing radiation [4. MRI on the other hand does not have this problem and will also allow a visualization of the adipose tissue.

The potential problems with MRI measures are linked to the technique by which images are obtained. MRI does not have the advantage of CT in terms of 
direct classification of tissues based on Hounsfield units and will therefore usually require an experienced professional to visually mark and measure the different tissues on each image making it a time consuming and expensive technique.

The development of a robust and accurate method for unsupervised segmentation of visceral and subcutaneous adipose tissue would be a both inexpensive and fast way of assessing abdominal fat.

The validation of MRI to assess adipose tissue has been done by [5]. A high correlation was found between adipose tissue assessed by segmentation of MR images and dissection in human cadavers. A number of approaches have been developed for abdominal assessment of fat by MRI. A semi automatic method that fits Gaussian curves to the histogram of intensity levels and uses manual delineation of the visceral area has been developed by [6] [ 7] uses fuzzy connectedness and Voronoi diagrams in a semi automatic method to segment adipose tissue in the abdomen. An Unsupervised method has been developed by [8] using active contour models to delimit the subcutaneous and visceral areas and fuzzy c-mean clustering to perform the clustering. 9 has developed an unsupervised method for assessment of abdominal fat in minipigs. The method performs a bias correction on the MR data and uses active contour models and dynamic programming to delimit the subcutaneous and visceral regions.

In this paper we present an unsupervised method that is robust to the poor image quality and large bias field that is present on older low field scanners. The method features a low number of parameters that are all non critical and give good results over a wide range of values. This is opposed to active contour models where accurate parameter tuning is required to yield good results. Furthermore, active contour models are not robust to large variations in intensity levels.

\section{Data}

The test data consisted of MR images from 300 subjects. The subjects were all human males with highly varying levels of obesity. Thus both very obese and very slim subjects were included in the data. Volume data was recorded for each subject in an anatomically bounded unit ranging from the bottom of the second lumbar vertebra to the bottom of the $5^{\text {th }}$ lumbar vertebra. In this unit slices were acquired with a spacing of $10 \mathrm{~mm}$. Only the T1 modality of the MRI data was used for further processing. A low field scanner was used for the image acquisition and images were scanned at a resolution of $256 \times 256$. The low field scanners generally have poor image quality compared to high field scanners. This is due to the presence of a stronger bias field and the extended amount of time needed for the image acquisition process thus not allowing breath-hold techniques to be used.

\section{Method}

\subsection{Bias Field Correction}

The slowly varying bias field present on all the MR images was corrected using a new way of sampling same tissue voxels evenly distributed over the subjects 
anatomy. The method works by first computing all local intensity maxima inside the subjects anatomy (the Region Of Interest - ROI) on a given slice. The ROI is then subdivided into a number of overlapping rectangular regions and the voxel with the highest intensity is stored for each region. We assume that this local maximum intensity voxel is a fat voxel. A threshold percentage is defined and all voxels with intensities below this percentage of the highest intensity voxel in each region is removed. We use a $85 \%$ threshold for all images. However, this parameter is not critical and equally good results are obtained over a range of values $(80-90 \%)$.

The dimensions of the regions are determined so that it is impossible to place such a rectangle within the ROI without it overlapping at least one high intensity fat voxel. We subdivide the ROI into 8 rectangles vertically and 12 rectangles horizontally for all images. Again these parameters are not critical and equally good results are obtained for subdivisions $6-10$ vertically and $6-12$ horizontally. The acquired sampling locations are spatially trimmed to get evenly distributed samples across the subjects anatomy.

We assume an image model where the observed original biased image is the product of the unbiased image and the bias field

$$
I_{\text {biased }}=I_{\text {unbiased }} \cdot \text { bias } .
$$

The estimation of the bias field was done by fitting a 3 dimensional Thin Plate Spline to the sampled points in each subject volume. We apply a smoothing spline penalizing bending energy.

Assume $N$ observations in $\mathbb{R}^{3}$, with each observation $\mathbf{s}$ having coordinates $\left[\begin{array}{lll}s_{1} & s_{2} & s_{3}\end{array}\right]^{T}$ and values $y$. Instead of using the sampling points as knots a regular grid of $n$ knots $\mathbf{t}$ is defined with coordinates $\left[\begin{array}{lll}t_{1} & t_{2} & t_{3}\end{array}\right]^{T}$. We seek to find a function $f$, that describes a 3-dimensional hypersurface that provides an optimal fit to the observation points with minimal bending energy. The problem is formulated as minimizing the function $S$ subject to $\mathrm{f}$.

$$
S(f)=\sum_{i=1}^{N}\left\{y_{i}-f\left(\mathbf{s}_{i}\right)\right\}^{2}+\alpha J(f)
$$

where $J(f)$ is a function for the curvature of $f$ :

$$
J(f)=\iiint_{\mathbb{R}^{3}} \sum_{i=1}^{3} \sum_{j=1}^{3}\left(\frac{\partial^{2} f}{\partial x_{i} x_{j}}\right)^{2} d x_{1} d x_{2} d x_{3}
$$

and $\mathrm{f}$ is of the form [10]:

$$
f(\mathbf{t})=\beta_{0}+\beta_{1}^{T} \mathbf{t}+\sum_{j}^{n} \delta_{j}\left\|\mathbf{t}-\mathbf{t}_{\mathbf{j}}\right\|^{3} .
$$

$\alpha$ is a parameter that penalizes for curvature. With $\alpha=0$ there is no penalty for curvature, this corresponds to an interpolating surface function where the 
function passes through each observation point. At higher $\alpha$ values the surface becomes more and more smooth since curvature is penalized. For $\alpha$ going towards infinity the surface will go towards the plane with the least squares fit, since no curvature is allowed.

To solve the system of equations we write the system on matrix form. First a coordinate matrix for the knots and the data points are defined.

$$
\begin{gathered}
\mathbf{T}_{\mathbf{k}}=\left[\begin{array}{ccc}
1 & \cdots & 1 \\
\mathbf{t}_{1} & \cdots & \mathbf{t}_{n}
\end{array}\right]_{[4 \times n]} \\
\mathbf{T}_{\mathbf{d}}=\left[\begin{array}{ccc}
1 & \cdots & 1 \\
\mathbf{s}_{1} & \cdots & \mathbf{s}_{N}
\end{array}\right]_{[4 \times N]} .
\end{gathered}
$$

Matrices containing all pairwise evaluations of the cubed distance measure from Equation 4 are defined as

$$
\begin{gathered}
\left\{\mathbf{E}_{\mathbf{k}}\right\}_{i j}=\left\|\mathbf{t}_{i}-\mathbf{t}_{j}\right\|^{3} \quad i, j=1, \cdots, n \\
\left\{\mathbf{E}_{\mathbf{d}}\right\}_{i j}=\left\|\mathbf{s}_{i}-\mathbf{t}_{j}\right\|^{3} \quad i=1, \cdots, N \quad j=1, \cdots, n
\end{gathered}
$$

$J(f)$ can be written as

$$
J(f)=\delta^{T} \mathbf{E}_{\mathbf{k}} \delta .
$$

We can now write equation 2 on matrix form, incorporating the constraints $\mathbf{T}_{k} \delta=\mathbf{0}$ by the method of Lagrange multipliers.

$$
S(f)=\left[\mathbf{Y}-\mathbf{E}_{\mathbf{d}} \delta-\mathbf{T}_{\mathbf{d}}^{T} \beta\right]^{T}\left[\mathbf{Y}-\mathbf{E}_{\mathbf{d}} \delta-\mathbf{T}_{\mathbf{d}}^{T} \beta\right]+\alpha \delta \mathbf{E}_{\mathbf{k}} \delta+\lambda^{T} \mathbf{T}_{\mathbf{k}} \delta
$$

where $\lambda$ is the Lagrange multiplier vector and $\beta=\left[\beta_{0} ; \beta_{1}\right]_{[4 \times 1]}$. By setting the 3 partial derivatives $\frac{\partial S}{\partial \delta}=\frac{\partial S}{\partial \beta}=\frac{\partial S}{\partial \lambda}=0$ we get the following linear system

$$
\left[\begin{array}{ccc}
\mathbf{E}_{\mathbf{d}}{ }^{T} \mathbf{E}_{\mathbf{d}}+\alpha \mathbf{E}_{\mathbf{k}} & \mathbf{E}_{\mathbf{d}}^{T} \mathbf{T}_{\mathbf{d}}{ }^{T} & \mathbf{T}_{\mathbf{k}}^{T} \\
\mathbf{T}_{\mathbf{d}} \mathbf{E}_{\mathbf{d}} & \mathbf{T}_{\mathbf{d}} \mathbf{T}_{\mathbf{d}}^{T} & \mathbf{0} \\
\mathbf{T}_{\mathbf{k}} & \mathbf{0} & \mathbf{0}
\end{array}\right]\left[\begin{array}{c}
\delta \\
\beta \\
\lambda
\end{array}\right]=\left[\begin{array}{c}
\mathbf{E}_{\mathbf{d}}{ }^{T} \mathbf{Y} \\
\mathbf{T}_{\mathbf{d}} \mathbf{Y} \\
\mathbf{0}
\end{array}\right] .
$$

An example result of the bias correction can be seen on Fig. 1,
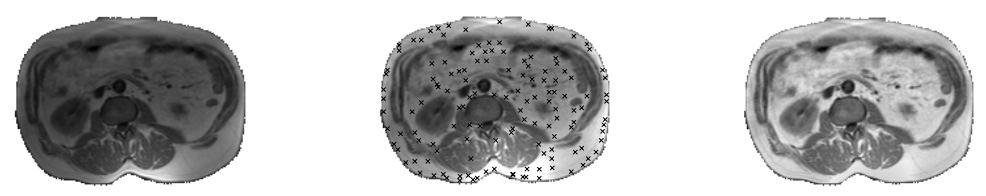

Fig. 1. (right) The MR image before the bias correction. (center) The sample points from which the bias field is estimated. (left) The MR image after the bias correction. 


\subsection{Identifying Image Structures}

Automatic outlining of 3 image structures was necessary in order to determine the regions for subcutaneous adipose tissue (SAT) and visceral adipose tissue (VAT): The external SAT outline, the internal SAT outline and the VAT area outline. First, a rough identification of the location of each outline was found using an active shape model trained on a small sample. Outlines found using this rough model were then used as constraints to drive a simple dynamic programming through polar transformed images.

\subsection{Active Shape Models}

The Active Shape Models approach developed by [12] is able to fit a point model of an image structure to image structures in an unknown image. The model is constructed from a set of $112 \mathrm{D}$ slices from different individuals at different vertical positions. This training set consists of images selected to represent the variation of the image structures of interest across all data. We have annotated the outer and inner subcutaneous outlines as well as the posterior part of the inner abdominal outline with a total of 99 landmarks. Fig. 2 shows an example of annotated images in the training set.
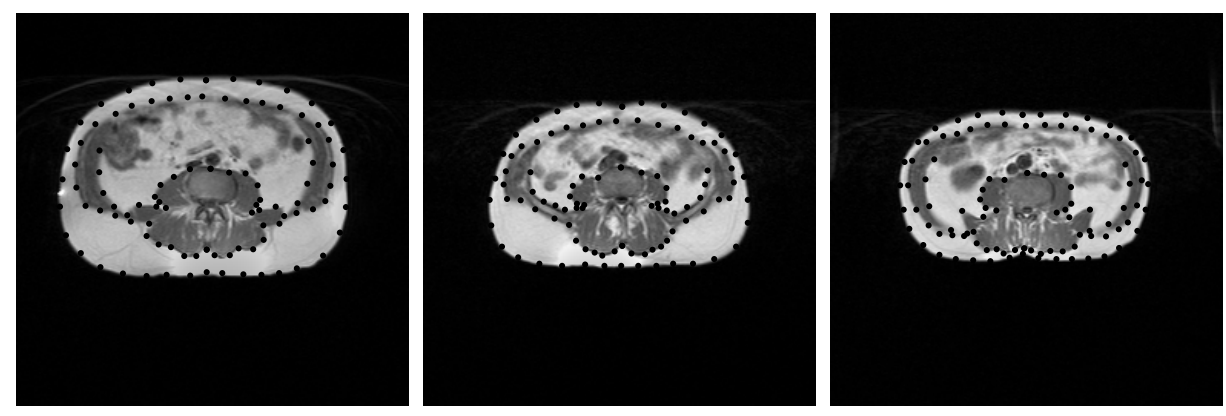

Fig. 2. 3 examples of annotated images from the training set

The 3 outlines are jointly aligned using a generalized Procrustes analysis [1314], and principal components accounting for $95 \%$ of the variation are retained.

The search for new points in the unknown image is done by searching along a profile normal to the shape boundary through each shape point. Samples are taken in a window along the sampled profile. A statistical model of the grey-level structure near the landmark points in the training examples is constructed. To find the best match along the profile the Mahalanobis distance between the sampled window and the model mean is calculated. The Mahalanobis distance is linearly related to the $\log$ of the probability that the sample is drawn from a Gaussianmodel. The best fit is found where the Mahalanobis distance is lowest and thus the probability that the sample comes from the model distribution is highest. 


\subsection{Dynamic Programming}

The shape points acquired from the active shape models were used as constraints for dynamic programming. First a polar transformation was applied to the images to give the images a form suitable for dynamic programming [15. A difference filter was applied radially to give edges from the original image a ridge representation in the transformed image. The same transformation was applied to the shape points of the ASM. The shape points were then used as constraints for the optimal path of the dynamic programming, only allowing the path to pass within a band of width 7 pixels centered on the ASM outline.

The optimal paths were then transformed back into the original image format to yield the outline of the external SAT border, the internal SAT border and the VAT area border. The method is illustrated on Fig. 3 .
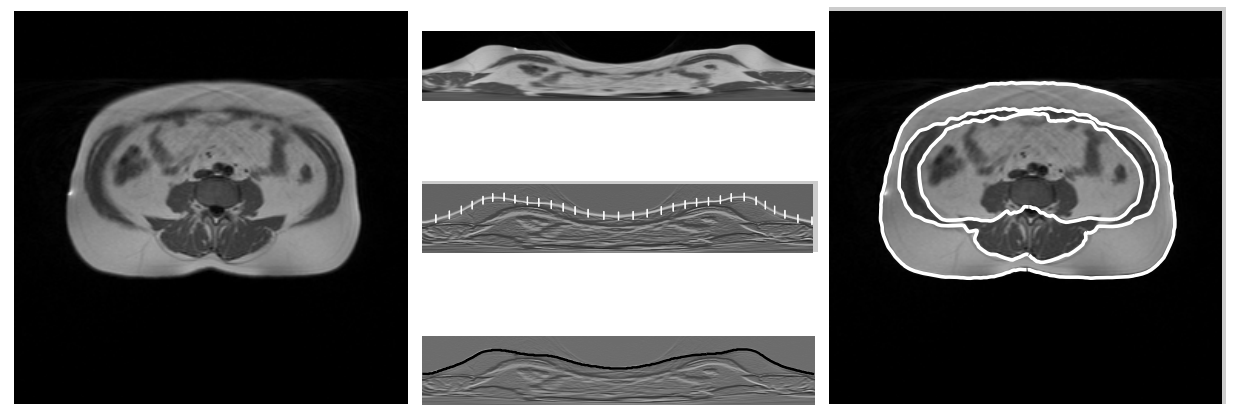

Fig. 3. Dynamic programming with ASM acquired constraints. (left) The bias corrected MR image. (center top) The polar transformed image. (center middle) The vertical difference filter applied on the transformed image with the constraint ranges superimposed (in white). (center bottom) The optimal path (in black) found through the transformed image for the external SAT border. (right) The 3 optimal paths from the constrained dynamic programming superimposed on the bias corrected image.

\subsection{Post Processing}

A set of voxels were defined for each of the 3 image structure outlines and set operations were applied to form the regions for SAT and VAT. Fuzzy c-mean clustering was used inside the VAT area to segment adipose tissue from other tissue. 3 classes were used: one for adipose tissue, one for other tissue and one for void. The class with the highest intensity voxels was assumed to be adipose tissue. Finally the connectivity of adipose tissue from the fuzzy c-mean clustering was used to correct a number of minor errors in regions where no clear border between SAT and VAT was available. A few examples of the final segmentation can be seen on Fig. 4 .

\section{Results}

The amount of voxels in each class for each slice in the subjects were counted and measures for the total volume of the anatomically bounded unit were calculated. 

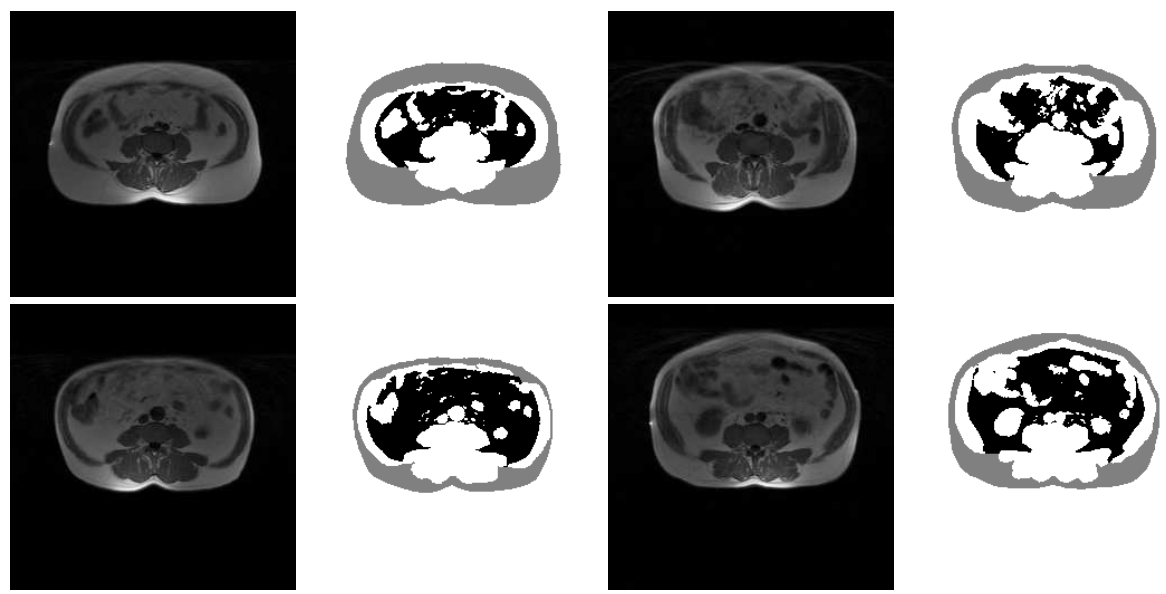

Fig. 4. 4 examples of the final segmentation. The segmented image is shown to the right of the original biased image. Grey: SAT; black:VAT; White:Other.

For each subject the distribution of tissue on the 3 classes: SAT, VAT and other tissue was computed. The results of the segmentation have been assessed by medical experts on a smaller subset of data and no significant aberrations between manual and unsupervised segmentation were found.

The unsupervised method was compared with manual segmentation. The manual method consist of manually segmenting the SAT by drawing the outlines of the internal and external SAT outlines. The VAT is estimated by drawing an outline around the visceral area and setting an intensity threshold that separates adipose tissue from muscle tissue.

A total of 14 subject volumes were randomly selected and segmented both automatic and manually. The correlation between the unsupervised and manual segmentation is high for both VAT $(r=0.9599, P<0.0001)$ and $\operatorname{SAT}(r=$ $0.9917, P<0.0001)$.

Figure $5(\mathrm{a})$ shows the Bland-Altman plot for SAT. The automatic method generally slightly overestimates compared to the manual method. The very blurry area near the umbilicus caused by the infeasibility of the breath-hold technique will have intensities that are very close to the threshold intensity between muscle and fat. This makes very slight differences between the automatic and manual threshold have large effects on the result.

The automatic estimates of the VAT also suffers from overestimation compared to the manual estimates, as seen on Figure $5(\mathrm{~b})$. The partial volume effect is particularly significant in the visceral area and the adipose tissue estimate is thus very sensitive to small variations of the voxel intensity classification threshold.

Generally, the main source of disparity between the automatic and manual methods is the difference in the voxel intensity classification threshold. The manual method generally sets the threshold higher than the automatic method, which causes the automatic method to systematically overestimate compared to the manual method. 

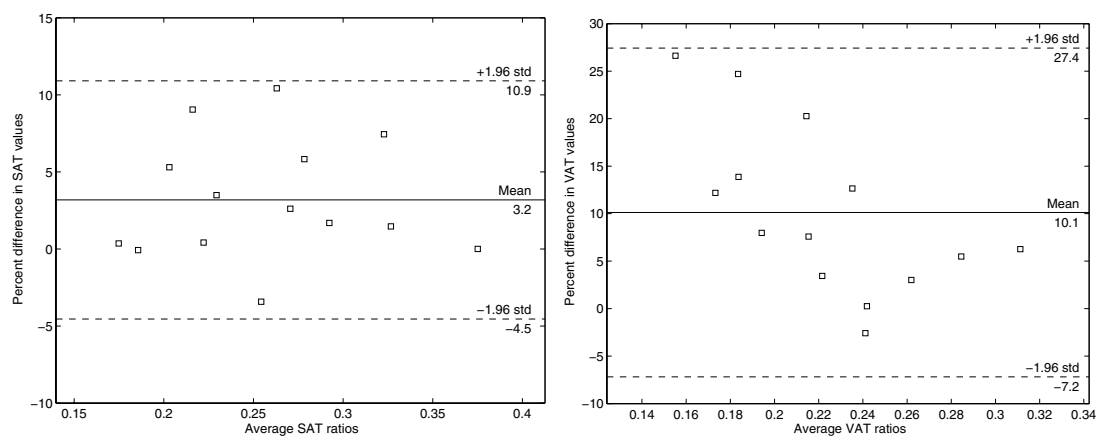

Fig. 5. (Left) Bland-Altman plot for SAT estimation on 14 subjects. (Right) BlandAltman plot for VAT estimation on 14 subjects.

Fat in the visceral area is hard to estimate due to the partial volume effect. The manual estimate might thus not be more correlated with the true amount of fat in the region than the automatic estimate. The total truncus fat on the 14 subjects was estimated using DEXA and the correlation was found to be higher between the estimated total fat of automatic segmentation $(r=0.8455)$ than the manual segmentation $(r=0.7913)$.

\section{Discussion}

The described bias correction procedure allows the segmentation method to be used on low field scanners. The method will improve in accuracy on images scanned by newer high field scanners with better image quality using the breathhold technique.

The use of ASM to find the general location of image structures makes the method robust to blurry areas (especially near the umbilicus) where a snake implementation is prone to failure 9. Our method yields good results even on images acquired over an extended time period where the breath-hold technique is not applied.

The combination of ASM with DP makes the method both robust and accurate by combining the robust but inaccurate high level ASM method with the more fragile but accurate low level DP method.

The method proposed here is fully automated and has a very low amount of adjustable parameters. The low amount of parameters makes the method easily adaptable to new data, such as images acquired from other scanners. Furthermore, all parameters yield good results over a wide range of values.

The use of an automated unsupervised method has the potential to be much more precise than manual segmentation. A large amount of slices can be analyzed at a low cost thus minimizing the effect of errors on individual slices. The increased feasible amount of slices to segment with an unsupervised method allows for anatomically bounded units to be segmented with full volume information. 
Using manual segmentation it is only feasible to segment a low number of slices in the subjects anatomy. The automatic volume segmentation will be less vulnerable to varying placement of organs on specific slices that could greatly bias single slice adipose tissue assessments. Furthermore the unsupervised segmentation method is not affected by intra- or inter-observer variability.

In conclusion, the presented methodology provides a both robust and accurate segmentation with only a small number of easily adjustable parameters.

Acknowledgements. We would like to thank Torben Leo Nielsen, MD Odense University Hospital, Denmark for allowing us access to the image data from the Odense Androgen Study and for valuable inputs during the course of this work.

\section{References}

1. Vague, J.: The degree of masculine differentiation of obesity: a factor determining predisposition to diabetes, atherosclerosis, gout, and uric calculous disease. Obes. Res. 4 (1996)

2. Bjorntorp, P.P.: Adipose tissue as a generator of risk factors for cardiovascular diseases and diabetes. Arteriosclerosis 10 (1990)

3. McNeill, G., Fowler, P.A., Maughan, R.J., McGaw, B.A., Gvozdanovic, D., Fuller, M.F.: Body fat in lean and obese women measured by six methods. Prof. Nutr. Soc. 48 (1989)

4. Van der Kooy, K., Seidell, J.C.: Techniques for the measurement of visceral fat: a practical guide. Int. J. Obes. 17 (1993)

5. Abate, N., Burns, D., Peshock, R.M., Garg, A., Grundy, S.M.: Estimation of adipose tissue by magnetic resonance imaging: validation against dissection in human cadavers. Journal of Lipid Research 35 (1994)

6. Poll, L.W., Wittsack, H.J., Koch, J.A., Willers, R., Cohnen, M., Kapitza, C., Heinemann, L., Mödder, U.: A rapid and reliable semiautomated method for measurement of total abdominal fat volumes using magnetic resonance imaging. Magnetic Resonance Imaging 21 (2003)

7. Jin, Y., Imielinska, C.Z., Laine, A.F., Udupa, J., Shen, W., Heymsfield, S.B.: Segmentation and evaluation of adipose tissue from whole body MRI scans. In: Ellis, R.E., Peters, T.M. (eds.) MICCAI 2003. LNCS, vol. 2878, pp. 635-642. Springer, Heidelberg (2003)

8. Positano, V., Gastaldelli, A., Sironi, A.M., Santarelli, M.F., Lmobardi, M., Landini, L.: An accurate and robust method for unsupervised assessment of abdominal fat by MRI. Journal of Magnetic Resonance Imaging 20 (2004)

9. Engholm, R., Dubinskiy, A., Larsen, R., Hanson, L.G., Christoffersen, B.Ø.: An adipose segmentation and quantification scheme for the abdominal region in minipigs. In: International Symposium on Medical Imaging 2006, San Diego, CA, USA. The International Society for Optical Engineering, SPIE (February 2006)

10. Green, P.J., Silverman, B.W.: Nonparametric regression and generalized linear models, a roughness penalty approach. Chapman \& Hall, Boca Raton (1994)

11. Hastie, T., Tibshirani, R., Friedman, J.: The elements of statistical learning. Springer, Heidelberg (2001) 
12. Cootes, T.F., Taylor, C.J.: Statistical models of appearence for medical image analysis and computer vision. In: Proc. SPIE Medical Imaging (2001) (to appear)

13. Gower, J.C.: Generalized procrustes analysis. Psychometrika 40 (1975)

14. Ten Berge, J.M.F.: Orthogonal procrustes rotation for two or more matrices. Psychometrika 42 (1977)

15. Glasbey, C.A., Young, M.J.: Maximum a posteriori estimation of image boundaries by dynamic programming. Journal of the Royal Statistical Society - Series C Applied Statistics 51(2), 209-222 (2002) 